\title{
Detection of HER2 through Antibody Immobilization Is Influenced by the Properties of the Magnetite Nanoparticle Coating
}

\author{
Enrique Villegas-Serralta, ${ }^{1}$ Oscar Zavala, ${ }^{2}$ Israel Alejandro Flores-Urquizo, ${ }^{3}$ \\ Perla E. García-Casillas $\mathbb{D}^{1}{ }^{1}$ and Christian Chapa González $\mathbb{D}^{1}$ \\ ${ }^{1}$ Instituto de Ingeniería y Tecnología, Universidad Autónoma de Ciudad Juárez, Ciudad Juárez, CHIH, Mexico \\ ${ }^{2}$ Instituto de Ciencias Biomédicas, Universidad Autónoma de Ciudad Juárez, Ciudad Juárez, CHIH, Mexico \\ ${ }^{3}$ Facultad de Ciencias Químicas, Universidad Autónoma de Nuevo León, San Nicolás de los Garza, NL, Mexico \\ Correspondence should be addressed to Christian Chapa González; christian.chapa@uacj.mx
}

Received 28 July 2017; Revised 27 December 2017; Accepted 12 February 2018; Published 2 April 2018

Academic Editor: Jin-Ho Choy

Copyright (c) 2018 Enrique Villegas-Serralta et al. This is an open access article distributed under the Creative Commons Attribution License, which permits unrestricted use, distribution, and reproduction in any medium, provided the original work is properly cited.

Considerable effort has been focused on improving the control of size, shape, and surface modifications to detect proteins. The purpose of this study was to compare the efficiencies of aminosilane-coated magnetite (As-M) nanoparticles (NPs), dextran-coated magnetite nanoparticles (Dx-M), and bare nanoparticles for conjugating single-chain variable fragment antibodies (scFvs) with the aim of detecting the human epidermal growth factor receptor 2 (HER2) protein. Dx-M and As-M NPs were characterized using scanning electron microscopy, energy dispersive X-ray spectroscopy, X-ray diffraction, and Raman spectroscopy. Dx-M and As-M were conjugated with a monoclonal scFv for active targeting of the HER2 antigen. Aminosilane surface coating enhanced the scFv conjugation efficiency over twofold compared to that of the dextran-coated magnetite NPs for the detection of HER2 proteins.

\section{Introduction}

The development of nanomaterials has been widely touted as a revolutionary paradigm shift for biological applications. In recent years, considerable effort has been focused on improving the control of size, shape, and surface modifications to gain better behavior as well as improved functionalization. Magnetic nanoparticles (NPs) have become widely studied due to the rapid increase in the number of applications in the biomedical field, such as magnetic resonance imaging [1], hyperthermia [2], drug delivery $[3,4]$, gene therapy $[5,6]$, protein immobilization $[7,8]$, and immunoassay [9]. Among other applications of magnetic NPs, for the immunoassay, the conjugation of NPs with antibodies is the critical step and severely limited by low efficiency of antibodies grafted on the NP surface.

Although there has been significant work done on the conjugation of chemically synthesized magnetite NPs to antibodies [10-15] there have been relatively few studies comparing the influence of antibody immobilization method in effectiveness of antigen detection. The conjugation of the proteins with magnetic NPs can be done via physical adsorption using polymer coatings, such as dextran, and by covalent immobilization using amino derivatives, such as aminosilane [3]. Dextran is a natural polysaccharide that confers exceptionally high colloidal stability to NPs [16]. 3-(2aminoethylamino) propyltrimethoxysilane (APTES) is the most frequently used compound to produce the aminosilane coating to enhance protein adhesion [17-20]. Both dextran and aminosilane have been used for the surface modification of NPs to immobilize antibodies and to improve the dispersion of magnetite NPs. Despite the influence on dispersion stability of the NPs, the type of coating has a remarkable influence on the effectiveness of antibody immobilization.

The effectiveness of conjugation has an impact on the detection of bioanalytes, such as cancer biomarkers. Overexpression of human epidermal growth factor receptor 2 (HER2) is common to several types of human carcinomas [21-23], is 
involved in cell growth, and is a recognized biomarker for breast cancer therapy [24-27]. The aim of this study is to compare aminosilane-coated magnetite (As-M) NPs and dextrancoated magnetite (Dx-M) NPs conjugated with recombinant single-chain variable fragment antibodies (scFvs) to Her2 (anti-HER2) through an adapted enzyme-linked immunosorbent assay (ELISA) in order to choose the most efficient scFv immobilizing agent.

\section{Materials and Methods}

2.1. Synthesis of Magnetite NPs. Magnetite NPs were synthesized by alkaline coprecipitation of two equivalents of ferric chloride $\left(\mathrm{FeCl}_{3} \cdot 6 \mathrm{H}_{2} \mathrm{O}\right.$; Mallinckrodt) and one equivalent of ferrous sulfate $\left(\mathrm{FeSO}_{4} \cdot 7 \mathrm{H}_{2} \mathrm{O}\right.$; J.T.Baker $)$ in $30 \%$ ammonium hydroxide solution $\left(\mathrm{NH}_{4} \mathrm{OH}\right.$; J.T.Baker) with stirring. The obtained precipitate was centrifuged at $6,000 \mathrm{rpm}$ and washed several times with distilled water until a $\mathrm{pH} \sim 7$ was reached. Finally, the precipitate was dried at $80^{\circ} \mathrm{C}$ for 24 hours.

2.1.1. scFv Conjugation on Dx-M NPs. For the preparation of Dx-M NPs, dextran (molecular weight 20,000 Da) was dissolved in distilled water. $\mathrm{FeCl}_{3}(0.01 \mathrm{M})$ and $\mathrm{FeSO}_{4}(0.005 \mathrm{M})$ were dispersed in $0.05 \%$ dextran solution. Synthesis of the NPs was done as previously described. The products were separated with a magnet and washed several times with deionized water. Dx-M was resuspended in phosphate buffer solution (pH 7.4). Then, $500 \mu \mathrm{L}$ of Dx-M suspension and $500 \mu \mathrm{L}$ of scFvs were mixed by stirring gently and incubated in a cold room at $4^{\circ} \mathrm{C}$ for 24 hours. The products were collected with a magnet and washed with buffer.

2.1.2. $s c F v$ Conjugation on As-M NPs. The obtained magnetite powder $(200 \mathrm{mg})$ was dispersed in $150 \mathrm{~mL}$ of ethanol and sonicated for 15 minutes, before the addition of 3-(2-aminoethylamino) propyltrimethoxysilane (AEPTMS, Sigma-Aldrich). After ultrasonic agitation for $30 \mathrm{~min}$, the suspended NPs were collected by magnetic decantation. The precipitated product (As-M) was washed with ethanol five times and dried at $80^{\circ} \mathrm{C}$ for 24 hours. The As-M NPs were resuspended in $1.0 \mathrm{~mL}$ of $2 \%$ glutaraldehyde aqueous solution with stirring for 4 hours at room temperature. Then, a mixture of $500 \mu \mathrm{L}$ of As-M and $500 \mu \mathrm{L}$ of scFvs was added and incubated in a cold room at $4^{\circ} \mathrm{C}$ for 24 hours to allow conjugation to take place. Finally, the solution was dialyzed with phosphate buffered saline (PBS).

2.2. Characterization of NPs. The bare magnetite sample was analyzed in PANalytical X'Pert MRD PRO device with a $\mathrm{Cu}-\mathrm{k} \alpha$ source $(\lambda=1.5406 \AA)$ operating at $40 \mathrm{kV}$ and $30 \mathrm{~mA}$ and at a scanning rate of $0.1^{\circ} 2 \theta \mathrm{s}^{-1}$ from 10 to $80^{\circ} 2 \theta$. Distances between peaks were compared to the International Centre for Diffraction Data JCDPS, number 5-0664. The average diffracting crystallite size of the prepared NPs was calculated using Scherrer's equation from the most intense peak. Scanning electron microscopy (SEM) was performed using a JEOL JSM6010LV/PLUS SEM to determine the particle size and morphology of the magnetite nanoparticles. For particle size analysis, the image processing software Scandium was used and 100 particles were analyzed to calculate the mean diameter \pm standard deviation (SD). SEM images were made with an accelerating voltage of $20 \mathrm{kV}$ and a working distance of $410 \mathrm{~mm}$. Energy dispersive Xray spectroscopy (EDS) using an Inca apparatus (Oxford Instruments, Oxford, UK) was done for elemental analysis or chemical characterization of the samples. TEM data was also provided to confirm the chemically well-defined magnetite nanoparticles. Hydrodynamic diameters and zeta potentials were analyzed using a dynamic light scattering (DLS) (Nanotrac Wave, Microtrac) operating at $25^{\circ} \mathrm{C}$ in a scattering angle of $180^{\circ}$; samples were suspended in Triton $2 \%, \mathrm{pH} \mathrm{7}$, and sonicated 5 minutes prior to measurement. Fourier transform infrared spectra (FTIR) were recorded using a spectrometer (Nicolet 6700/Thermo Electron) to identify functional groups present in the surface-modified magnetite nanoparticles. Infrared spectra with attenuated total reflection (ATR) were recorded with a resolution of $4 \mathrm{~cm}^{-1}$ and the scan range was set from 4000 to $600 \mathrm{~cm}^{-1}$. Raman spectra were recorded in a WiTec system model Alpha 300 RA equipped with a $785 \mathrm{~nm}$ laser source. Raman spectroscopy was used to measure bands of -S-S-, -N-C-N-, and -C-S-, which are likely to be found in proteins (scFvs). These groups are rich in $\pi$-electron and, thus, have larger polarizability and totally symmetric vibrational modes that are Raman active but may not be infrared (IR) active and show no IR bands [28]. Because of these differences and considering that water, the natural medium for proteins is a weak Raman scatter; Raman was the method chosen for characterization of antibodyNP conjugate. In addition, for proteins, studies focused on investigating Raman spectra of proteins and the successful design of NP probes has allowed advances in immunoassays [29].

2.3. ELISA. Aliquots $(50 \mu \mathrm{L})$ of the homogenized suspensions (scFv-Dx-M, scFv-As-M, and Dx-M) were deposited on the bottom of wells in a 96 well microplate. Next, a permanent magnet was used to immobilize the conjugates at the bottom. The plates were blocked with PBS containing $5 \%$ bovine serum albumin (BSA). Successively, anti-HA antibody and horseradish peroxidase (HRP; GE Healthcare Life Sciences) were added and incubated at $37^{\circ} \mathrm{C}$ for 1 hour. Unbound anti-HA was discarded by washing. All washing steps were performed with $200 \mu \mathrm{L}$ of PBS- $0.05 \%$ Tween 20. Finally, $50 \mu \mathrm{L}$ of 2,2' -Azino-bis(3-ethylbenzthiazoline-6sulfonic acid) (ABTS, Roche) was added and incubated for 15 minutes at room temperature and the absorbance was read at $\lambda=405 \mathrm{~nm}$ in a Benchmark Plus device (Bio-Rad, Hercules, CA, USA). Each independent experiment was run in triplicate.

2.4. Statistical Analyses. Data are presented as the mean \pm standard deviation (SD). Differences between groups were assessed by one-way analysis of variance (ANOVA) followed by Tukey's Multiple Comparison Analysis test. Values were determined as significant when $P<0.05$. 


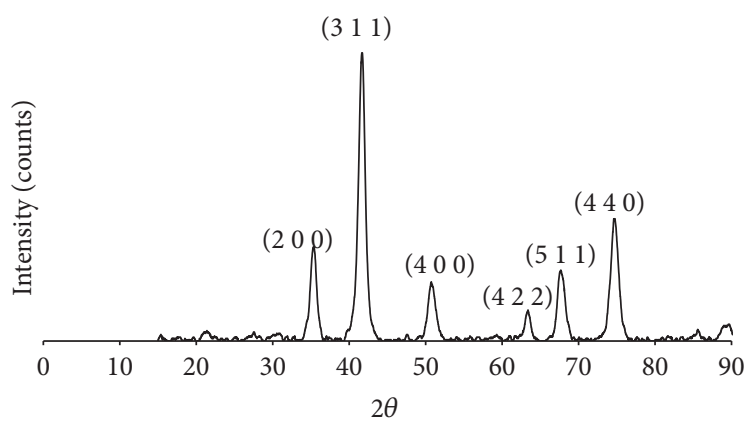

FIGURE 1: XRD pattern for the magnetite $\left(\mathrm{Fe}_{3} \mathrm{O}_{4}\right)$ nanoparticles.

\section{Results and Discussion}

The X-ray diffraction (XRD) pattern of uncoated magnetite is shown in Figure 1, with the peaks corresponding to the planes for (2 20 ), (3 11 1), (4 00 ) , (4 $\left.\begin{array}{lll}4 & 2\end{array}\right),\left(\begin{array}{lll}5 & 1 & 1\end{array}\right)$, and (4 4 0 ); peaks are matched in position and in relative intensity to the inverse spinel structure of magnetite pattern according to JCPDS 5-0664. The average size of the crystallite was calculated by the Scherrer equation [30-33], in which peak width is inversely proportional to crystallite size. The average crystallite size was $10.1 \mathrm{~nm}$, which is consistent with the size of superparamagnetic particles [34].

SEM revealed the morphological properties of the prepared samples (Figure 2). Monodisperse magnetite NPs were successfully synthesized. While a number of suitable methods have been developed for the synthesis of magnetic NPs, the coprecipitation method yielded magnetite NPs with a relatively uniform and spherical morphology with narrow particle size distribution $(8 \pm 5 \mathrm{~nm})$ according to SEM measurements. The mean diameter of both coated particles was around $20 \mathrm{~nm}$. Furthermore, the EDS spectrum revealed $\mathrm{Fe}$ and $\mathrm{O}$ in bare magnetite samples; $\mathrm{Fe}, \mathrm{O}$, and $\mathrm{C}$ in $\mathrm{Dx}-$ $\mathrm{M}$ samples; and $\mathrm{Fe}, \mathrm{O}$, and $\mathrm{Si}$ in As-M samples, consistent with the presence of aminosilane-modified NPs. In all the samples, the carbon signal appeared due to the preparation of the sample; however in Dx-M sample the signal was more intense.

Representative TEM image with corresponding size distribution of magnetite nanoparticles is shown in Figure 3. Well-defined nearly spherically shaped nanoparticles were indeed obtained. Their average size, determined from the statistical sample of over one hundred nanoparticles, was found to be $9.0 \mathrm{~nm}$, while standard deviation of $2.3 \mathrm{~nm}$ indicated a reasonably narrow size distribution. Moreover, notice the accurate agreement between the average diameter assessed from both XRD and TEM data confirming the presence of monodomain nanoparticles.

Figure 4 shows the hydrodynamic size of $\mathrm{M}, \mathrm{As}-\mathrm{M}$, and Dx-M samples. The particle size, its distribution, and the surface charge of $\mathrm{Fe}_{3} \mathrm{O}_{4}$ are very important parameters not only for modifying $\mathrm{Fe}_{3} \mathrm{O}_{4}$ nanoparticles, but also for biomedical applications. The coating of the nanoparticles can be evaluated by measuring the zeta potential. The results of the mean hydrodynamic diameter, polydispersity index (PDI), and zeta potential of the surface-modified magnetite nanoparticles
TABLE 1: Hydrodynamic diameter, polydispersity index, and zeta potential of M, As-M, and Dx-M.

\begin{tabular}{lccc}
\hline Sample & HD, $\mathrm{nm}$ & SD & ZP, mV \\
\hline $\mathrm{M}$ & 4.97 & 1.64 & -1.10 \\
As-M & 11.48 & 5.68 & -23.10 \\
Dx-M & 7.47 & 2.31 & -8.56 \\
\hline
\end{tabular}

Abbreviations. M: magnetite nanoparticles; Dx-M: dextran-modified magnetite nanoparticles; As-M: AEPTMS-modified magnetite nanoparticles; HD: hydrodynamic diameter; SD: standard deviation; ZP: zeta potential.

developed in this study are listed in Table 1. The smallest hydrodynamic diameter of about $4.97 \pm 1.64 \mathrm{~nm}$ was obtained for bare magnetite nanoparticles. The mean particles size of As- $\mathrm{M}$ and $\mathrm{Dx}-\mathrm{M}$ was found to be11.48 \pm 5.68 , and $7.47 \pm$ $2.31 \mathrm{~nm}$, respectively, suggesting that the AEPTMS coating did induce a significant increase of the mean particles size $(P<0.05)$. Contrastingly, the coating with dextran did not induced a significant increase of the particles diameter $(P<$ $0.05)$. The zeta potential value obtained for $M$ was slightly negative $(-1.10 \mathrm{mV})$; this explains why magnetite nanoparticles do not remain suspended for a long period of time. A zeta potential of $-23.10 \mathrm{mV}$ was observed for magnetite nanoparticles coated with AEPTMS. Thus, the use of AEPTMS as a coating also contributed to an increase in colloidal stability. The zeta potential of Dx-M nanoparticles was $-8.56 \mathrm{mV}$, which suggested that the nanoparticles were mainly stabilized by steric repulsion.

The FTIR-ATR comparative spectra of bare and coated $\mathrm{Fe}_{3} \mathrm{O}_{4}$ nanoparticles, as well as the coating molecules, are displayed in Figure 5. The samples that contain magnetite (M, Dx-M, and As-M) clearly reveal the presence of strong IR absorption band between 500 and $600 \mathrm{~cm}^{-1}$ characteristic to the $\mathrm{Fe}-\mathrm{O}$ vibration. IR spectra of pure AEPTMS are shown in Figure 5(b) while the IR spectra of As-M are shown in Figure 5(c). The IR spectrum of AEPTMS exhibits absorptions at $2890 \mathrm{~cm}^{-1}$ and $1059 \mathrm{~cm}^{-1}$, which are characteristic peaks of the stretching vibration of $\mathrm{C}-\mathrm{H}$ and $\mathrm{Si}-\mathrm{O}$, respectively. In the spectrum for As-M the peaks for the functional group $\mathrm{N}-\mathrm{H}$ and silane binding are marked. A displacement of the Si-O peak is observed in the AsM NPs. The peak found at $1383 \mathrm{~cm}^{-1}$ attributed to the stretching vibrations of $\mathrm{C}-\mathrm{N}$ bond, along with the band at $3360 \mathrm{~cm}^{-1}$ attributed to the $\mathrm{H}-\mathrm{N}-\mathrm{H}$ bending mode of free amino groups, indicates that the AEPTMS has been grafted onto the nanoparticles' surface. In the same way, Figures 5(d) and 5(e) give the IR absorption spectra of pure dextran and $D x-M$, respectively. The very broad band at $3380 \mathrm{~cm}^{-1}$ in both Dx and Dx-M can be attributed to the stretching vibration of $\mathrm{O}-\mathrm{H}$. The absorption bands at $2924 \mathrm{~cm}^{-1}$ can be ascribed to the asymmetrical stretching vibration of $\mathrm{C}-\mathrm{H}$ and the band at $1008 \mathrm{~cm}^{-1}$ belongs to stretching vibrations of C-O-C bond and glycosidic bridge. Regardless of the $\mathrm{Fe}-\mathrm{O}$ band found in Dx-M, very few differences can be found in the IR spectra of Dx and Dx-M which indicates that dextran coating occurs by physical adsorption.

Nevertheless, to further evaluate the antibody Raman spectroscopy was conducted. Raman spectra of the obtained 


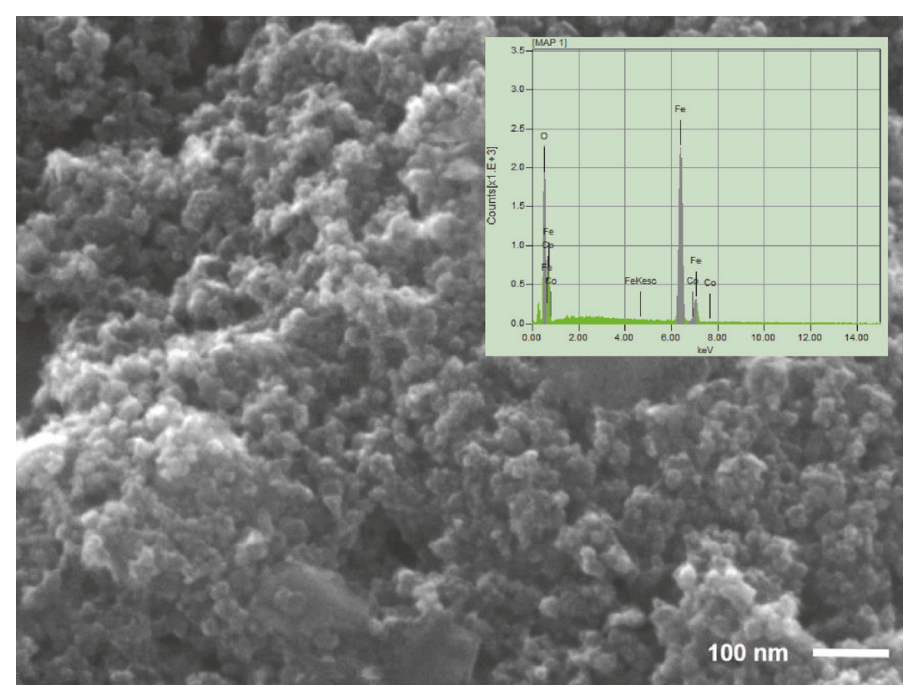

(a)

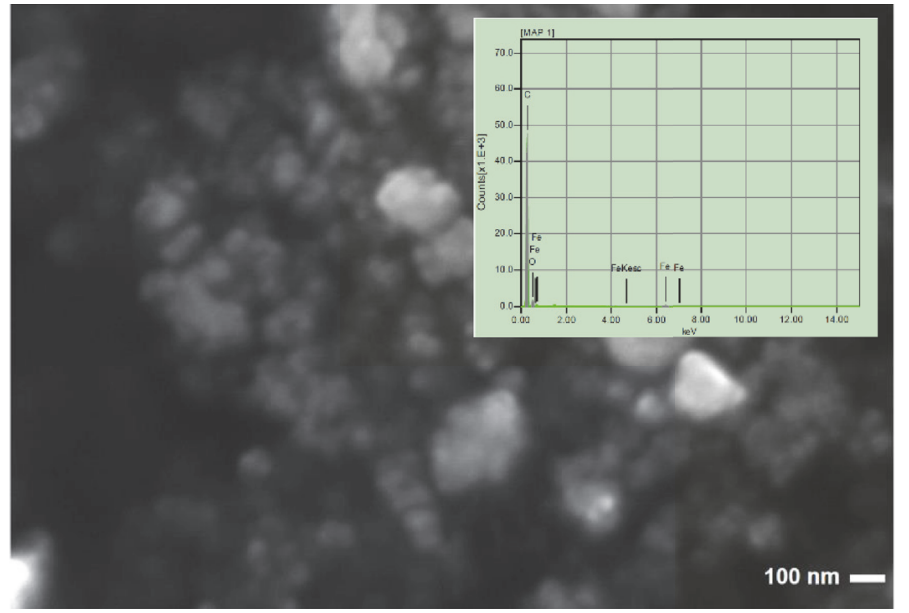

(b)

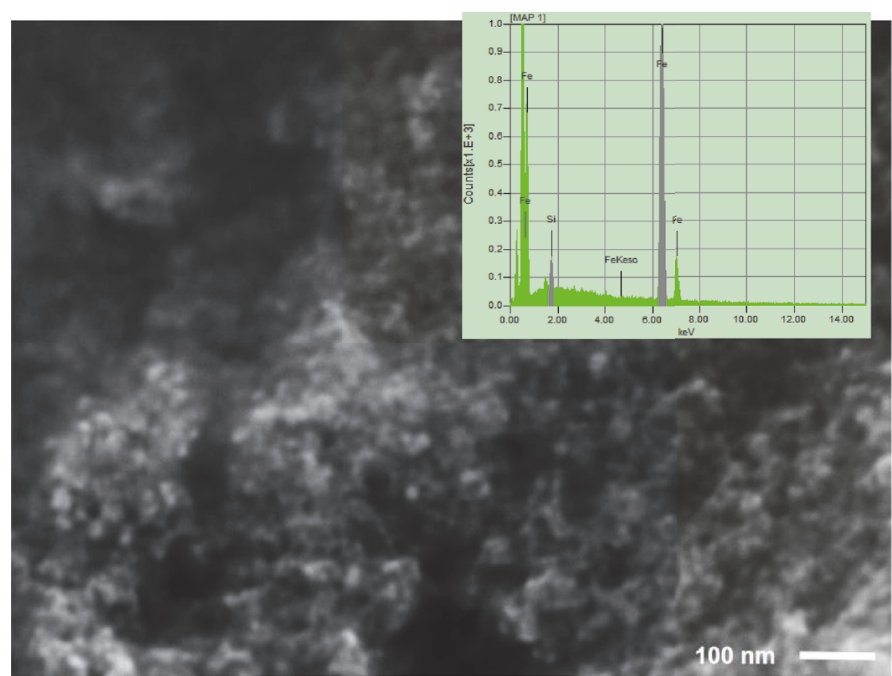

(c)

FIGURE 2: SEM image and EDS analysis of (a) uncoated magnetite nanoparticles, (b) DX-M nanoparticles, and (c) As-M nanoparticles. 


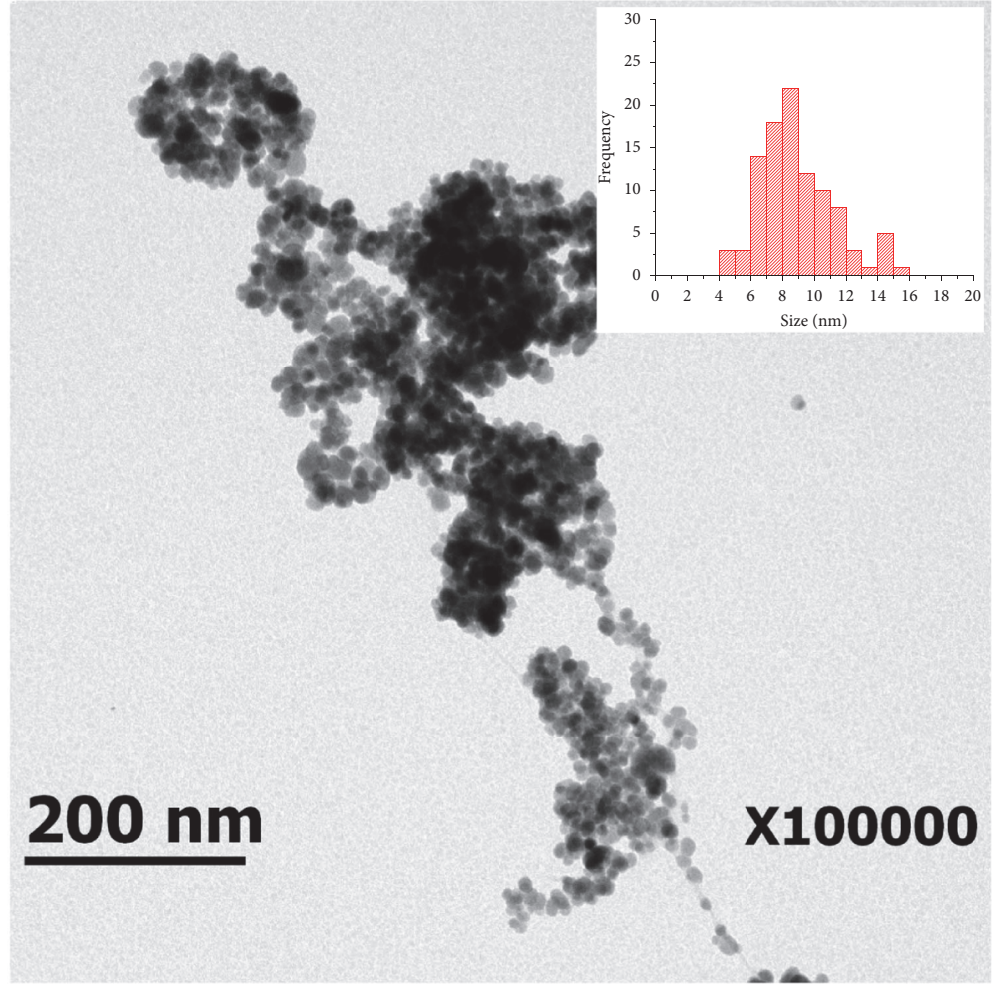

FIGURE 3: TEM image of bare magnetite nanoparticles with image with corresponding size distribution.

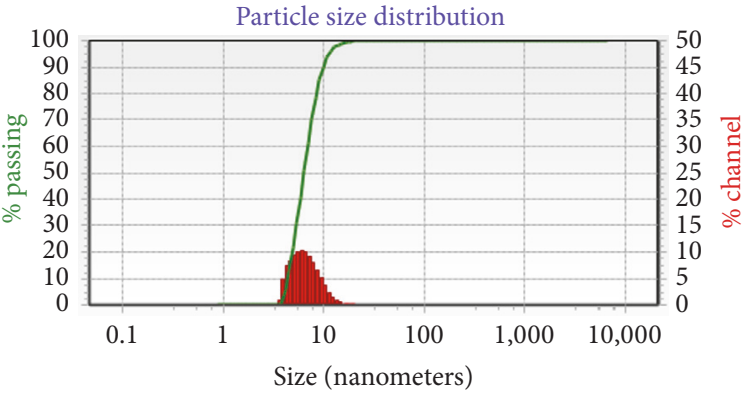

(a)

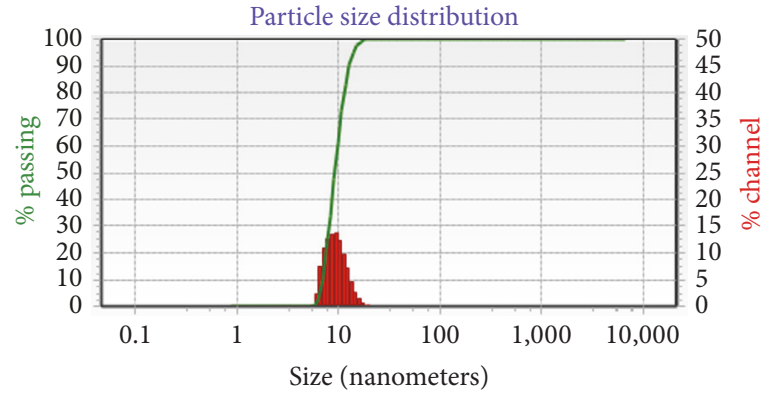

(b)

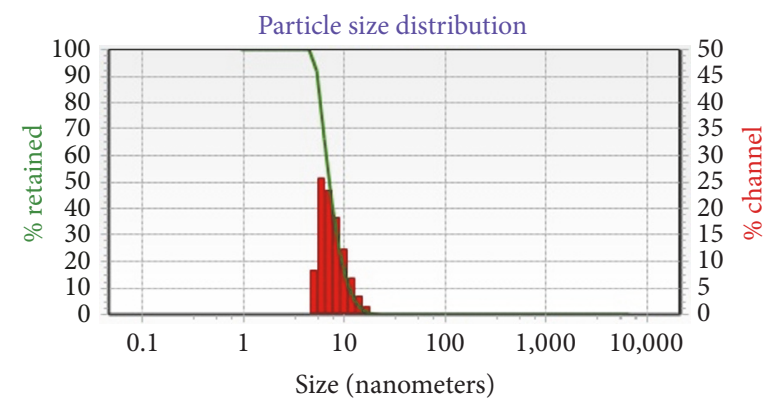

(c)

FIGURE 4: Particle size distribution of (a) magnetite, (b) AEPTMS-coated magnetite, and (c) dextran-coated magnetite. 


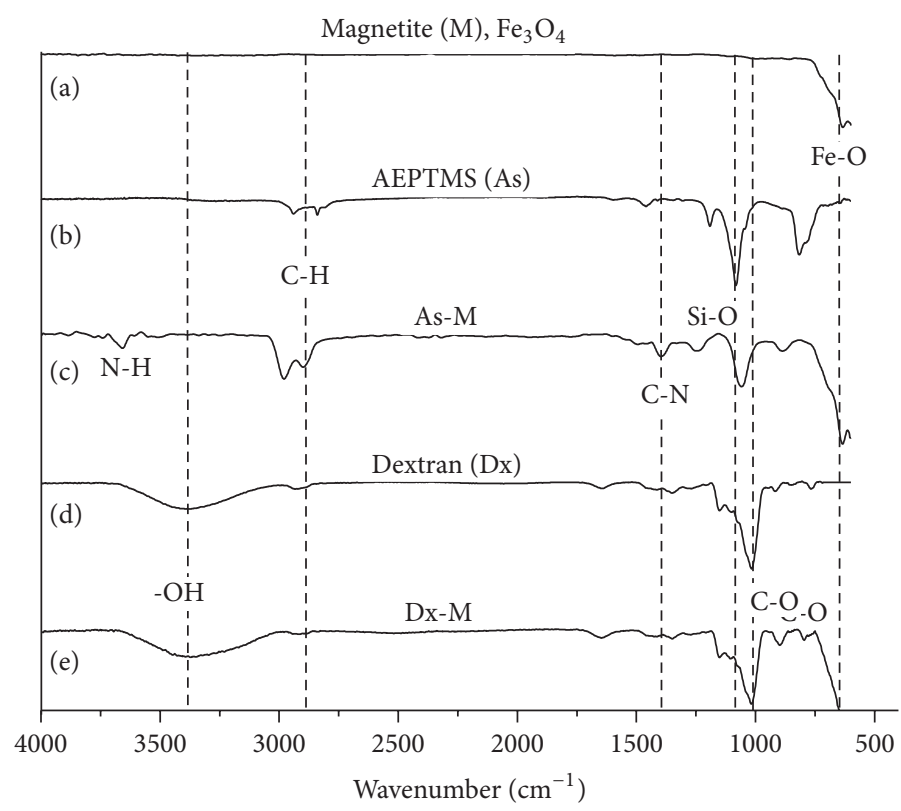

FIGURE 5: FTIR spectra of (a) magnetite, (b) AEPTMS, (c) aminosilane-coated magnetite, (d) dextran, and (e) dextran-coated magnetite.

materials are shown in Figure 6. According to the literature, the modes present at 207,392, and $470 \mathrm{~cm}^{-1}$ (Figure 6(a)) correspond to magnetite [35] and the band at near $1300 \mathrm{~cm}^{-1}$ is also attributable at magnetite $[36,37]$. In the other samples, the spectral bands seen below $800 \mathrm{~cm}^{-1}$ are primarily attributed to presence of magnetite [38] (Figures 6(b), 6(c), 6(e), and 6(f)). The Raman spectrum for the Dx-M included the typical signals for the dextran and weak bands typical of magnetite. For the Raman spectroscopy of the $\mathrm{Dx}-\mathrm{M}$, the surface modification of dextran-coated magnetite manifested in the electronic and vibrational properties of the magnetite and resulted in the lowering of the energy (Figure 6(b)). For aminosilane treated surfaces (Figure 6(c)), the characteristic bands at $1117 \mathrm{~cm}^{-1}$ corresponded to Si-OC, $1348 \mathrm{~cm}^{-1}$ could be attributed to $\mathrm{Csp}^{3}$ bound with or without heteroatoms, $1575 \mathrm{~cm}^{-1}$ was associated with bound $\mathrm{Csp}^{2}$, and $3300 \mathrm{~cm}^{-1}$ was related to an amine group. The results were consistent with the presence of aminosilane on the surface of the magnetite NPs. Raman spectroscopy of the antibody $(\mathrm{Ab})$ soluble scFv (Figure 6(d)) was obtained. The test solution contained $1 \mu \mathrm{g} / \mathrm{mL}$ scFv diluted in PBS. The Raman spectra scFv is typical of proteins $[38,39]$ with bands associated with disulfide bonds (-S-S-, $\left.544 \mathrm{~cm}^{-1}\right)$, polypeptide backbone $\left(\mathrm{C} \alpha-\mathrm{C}, 940 \mathrm{~cm}^{-1}\right)$, aromatic amino acids (phenylalanine, tryptophan, and tyrosine, bands from 1000 to $1205 \mathrm{~cm}^{-1}$ ), C-H deformation (1325 and $1452 \mathrm{~cm}^{-1}$ ), and amide III $\left(1267 \mathrm{~cm}^{-1}\right)$. The immobilization of the antibodies in both conjugates Dx-M and As-M were consistent with the Raman spectra of Dx-M-Ab (Figure 6(e)) and As$\mathrm{M}-\mathrm{Ab}$ (Figure 6(f)); both exhibited bands characteristic of the scFvs. The Raman spectrum for the Dx-M-Ab conjugates included the typical signals for the dextran and weak bands typical of proteins (amide I and phenylalanine aromatic ring near at $1600 \mathrm{~cm}^{-1}$ ). The Raman band at $\approx 1550 \mathrm{~cm}^{-1}$ arises from an indole ring vibration that is contributed mainly by Trp in Dx-M-Ab and As-M-Ab samples [20].

The conjugated product was verified by ELISA using a magnetic separator (Figure 7). Fifty microliters of the homogenized suspensions (scFv-Dx-M, scFv-As-M, and DxM) were used for ELISA using a magnet to immobilize the conjugates (Figure 7) using HRP-conjugated anti-HA. The ABTS assay is a colorimetric assay based on the ABTS cation radical formation. The radical formation is catalyzed by the reduction of HRP in the presence of $\mathrm{H}_{2} \mathrm{O}_{2}$. Employing this assay, we are able to compare the absorbance generating from the Dx-M-Ab and As-M-Ab conjugates and Dx-M nonconjugate $\mathrm{scFv}$ (as the negative control) against a secondary anti-HA antibody. The 2-fold difference in ELISA absorbance between scFv-AsM conjugates and scFv-DxM was indicative of successful conjugation (Figure 8). Based on the results of the evaluation of the ability of anti-HER2 scFvmagnetite conjugate in binding and detecting the studied protein, the chemical modification with aminosilane remarkably improved the protein detection. In addition, the success of binding to HER2 by As-M confirmed the existence of stable chemical bonding between the NPs and antibody. This was not evident in the Dx-M material whose modification occurs primarily by physical adsorption. However, a considerable difference was observed with respect to the first coating. The results allowed us to infer that, despite the washes performed on the aminosilane coating, the continuous antibody coupled to the nanoparticles and was recognized by the secondary anti-HA antibody. The results indicate that this coating is suitable for this type of material bioconjugate. 


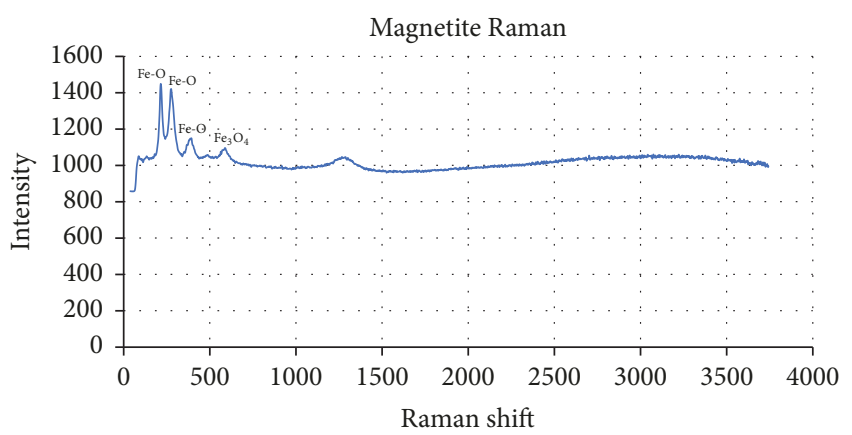

(a)

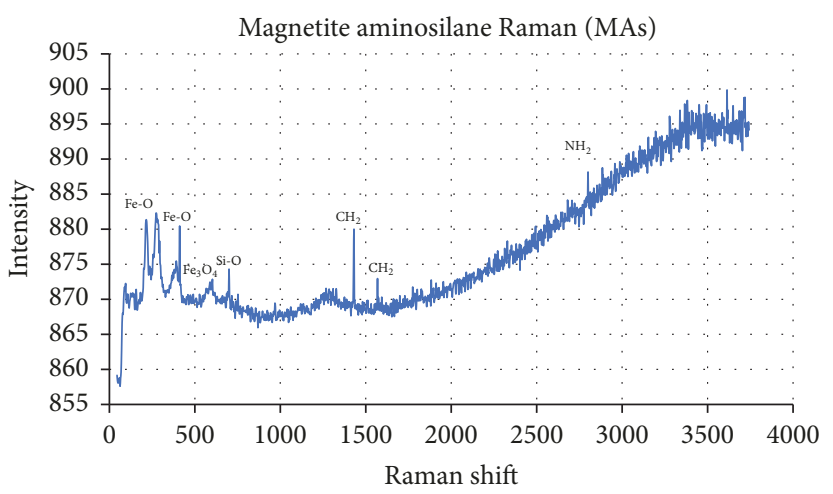

(c)

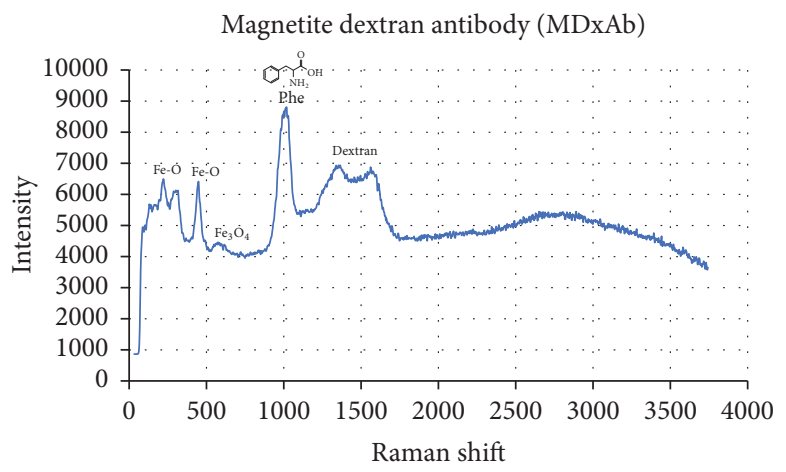

(e)

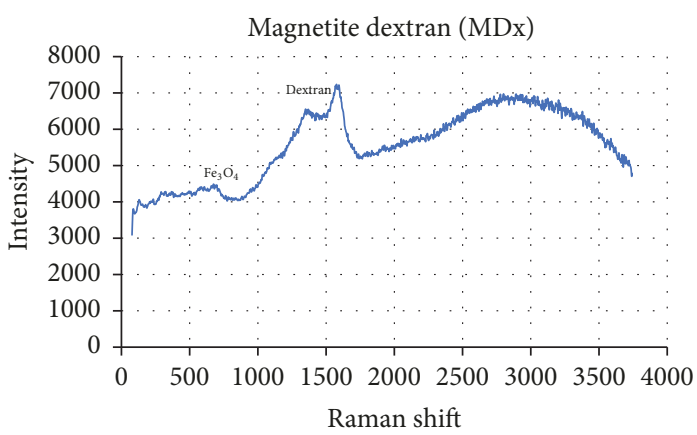

(b)

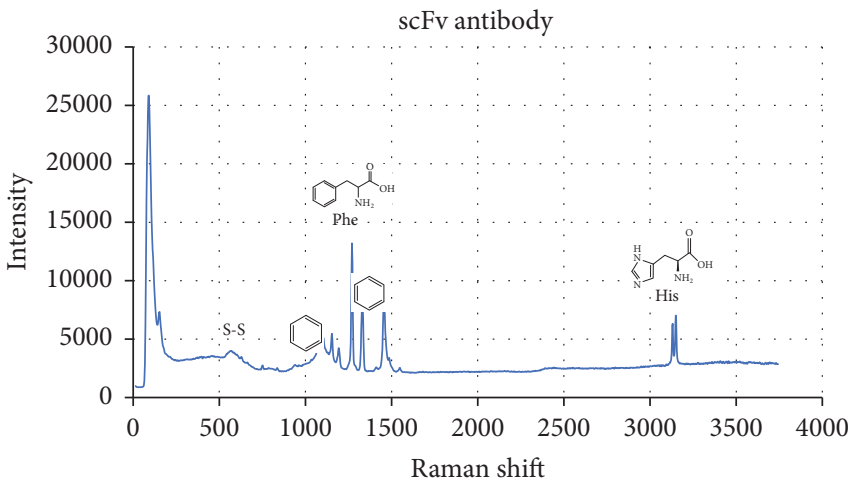

(d)

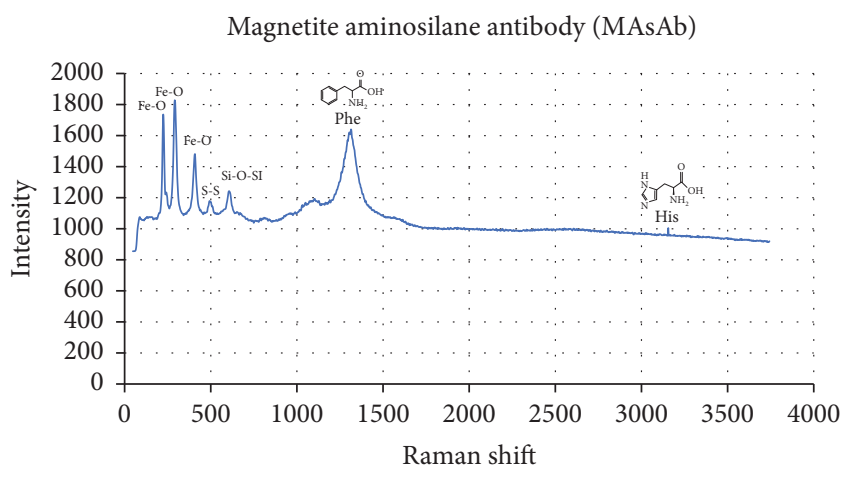

(f)

Figure 6: Comparative Raman spectra of (a) magnetite, (b) dextran-coated magnetite, (c) aminosilane-coated magnetite, (d) scFv antibody, (e) scFv conjugated with dextran-coated magnetite, and (f) scFv conjugated with aminosilane-coated magnetite.

\section{Conclusions}

In this study, well-defined magnetite nanoparticles with a narrow size distribution and coated with AEPTMS and dextran were successfully obtained. Furthermore, zeta potential measurements show that the magnetite nanoparticles coated with AEPTMS or dextran are stable in aqueous suspension at $\mathrm{pH} 7$ which makes these nanomaterials suitable for biomedical applications. Surface-modified magnetite nanoparticles containing antibodies were prepared and their antigen recognition ability toward HER2 as a model biomarker was performed. We successfully used this nanomaterial to study how coating changes the efficiency of antibody conjugation and, consequently, how it also affects protein detection in the magnetic bead ELISA. As-M NPs were more efficient in scFv immobilization than Dx-M NPs conjugated with recombinant $\mathrm{scFv}$ anti-Her2. This strategy provides an alternative approach to controlling surface functionalization with a view to preparing high-efficiency receptor for specific proteins. The immunoconjugate was proven to have a biomarkertargeting activity. Such As-M might be very useful for biomagnetically targeted detection in several types of human carcinoma.

\section{Conflicts of Interest}

The authors declare that there are no conflicts of interest regarding the publication of this article. 

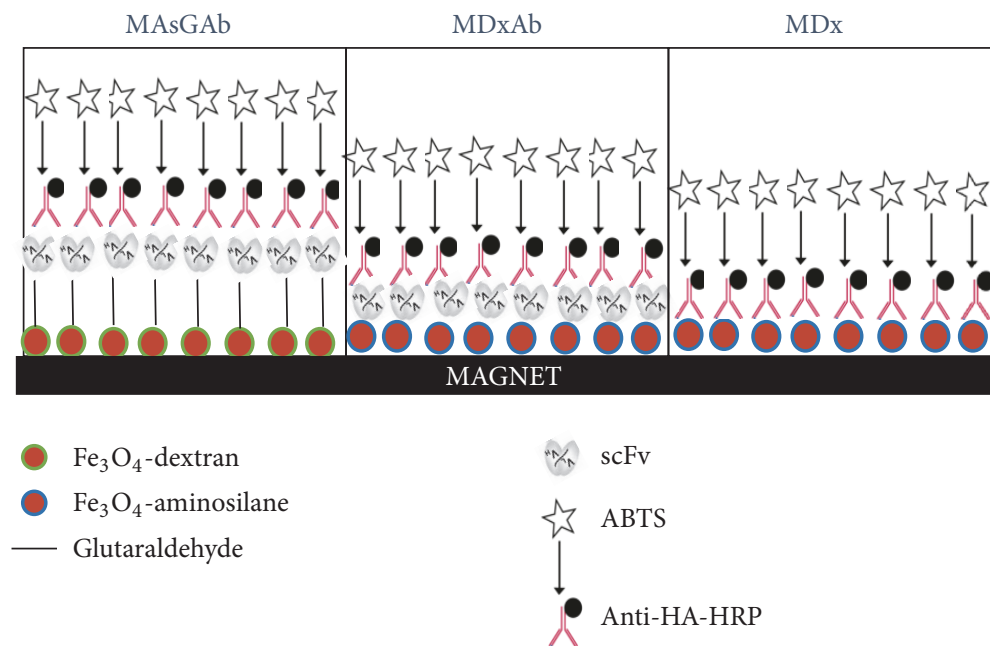

FIGURE 7: Scheme of the adapted ELISA using a permanent magnet to immobilize the conjugates.

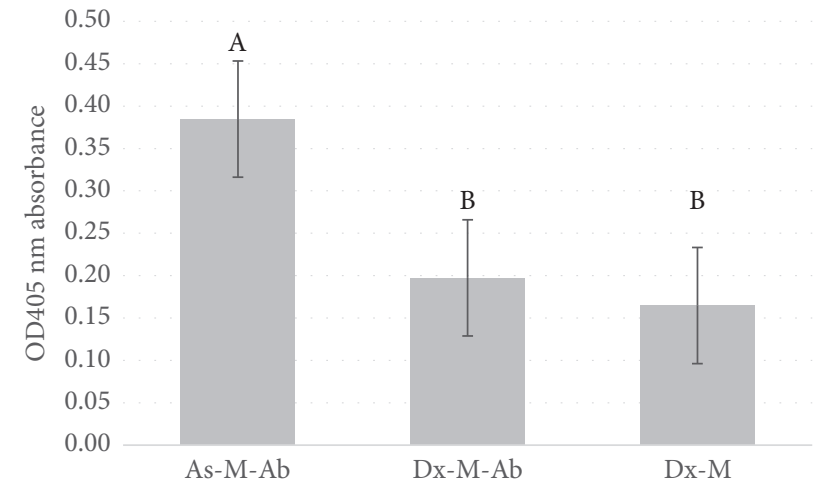

FIGURE 8: ELISA distinguishes specific binding to HER2 protein. All nanomaterial, except the As-M-antibody, has an absorbance value $<0.20$. Anti-HER2 scFv conjugated with As-M can detect HER2 protein, with a 1.95 -fold difference compared with Dx-Mantibody. Dx-M (nonconjugate scFv) was used as the negative control. The results were obtained from triplicate determinations and are expressed as mean $\pm \mathrm{SD}$.

\section{Acknowledgments}

The authors thank Karla Jacqueline Licona, for her help in preparing the manuscript, and Professor Carlos Rodriguez from UTCJ, for providing the SEM-EDS facilities.

\section{References}

[1] S. Shabestari Khiabani, M. Farshbaf, A. Akbarzadeh, and S. Davaran, "Magnetic nanoparticles: preparation methods, applications in cancer diagnosis and cancer therapy," Artificial Cells, Nanomedicine and Biotechnology, vol. 45, no. 1, pp. 6-17, 2017.

[2] K. Adhikary and M. Banerjee, "A thermofluid analysis of the magnetic nanoparticles enhanced heating effects in tissues embedded with large blood vessel during magnetic fluid hyperthermia," Journal of Nanoparticles, vol. 2016, 18 pages, 2016.

[3] L. Buzoglu, E. Maltas, M. Ozmen, and S. Yildiz, "Interaction of donepezil with human serum albumin on amine-modified magnetic nanoparticles," Colloids and Surfaces A: Physicochemical and Engineering Aspects, vol. 442, pp. 139-145, 2014.

[4] E. Maltas and M. Ozmen, "Spectrofluorometric and thermal gravimetric study on binding interaction of thiabendazole with hemoglobin on epoxy-functionalized magnetic nanoparticles," Materials Science and Engineering C: Materials for Biological Applications, vol. 54, pp. 43-49, 2015.

[5] J. Roacho-Perez, H. Gallardo-Blanco, M. Sanchez-Dominguez, P. Garcia-Casillas, C. Chapa-Gonzalez, and C. Sanchez-Dominguez, "Nanoparticles for death-induced gene therapy in cancer (Review)," Molecular Medicine Reports, 2017.

[6] E. Maltas, M. Ozmen, and H. Cingilli Vural, "Interaction of L-myc oncogene in breast cancer with irinotecan onto functionalized magnetic nanoparticles," Materials Letters, vol. 106, pp. 8-10, 2013.

[7] M. Bayrakci, O. Gezici, S. Z. Bas, M. Ozmen, and E. Maltas, "Novel humic acid-bonded magnetite nanoparticles for protein immobilization," Materials Science and Engineering C: Materials for Biological Applications, vol. 42, pp. 546-552, 2014.

[8] E. Maltas, M. Ozmen, H. C. Vural, S. Yildiz, and M. Ersoz, "Immobilization of albumin on magnetite nanoparticles," Materials Letters, vol. 65, no. 23-24, pp. 3499-3501, 2011.

[9] A. E. Urusov, A. V. Petrakova, A. V. Zherdev, and B. B. Dzantiev, "Magnetic nanopartices as carriers for immunoassays," Nano Hybrids and Composites, vol. 13, pp. 54-62, 2017.

[10] M. Suzuki, M. Shinkai, M. Kamihira, and T. Kobayashi, "Preparation and characteristics of magnetite-labelled antibody with the use of poly(ethylene glycol) derivatives," Biotechnology and Applied Biochemistry, vol. 21, pp. 335-45, 1995.

[11] E.-Q. Song, J. Hu, C.-Y. Wen et al., "Fluorescent-magneticbiotargeting multifunctional nanobioprobes for detecting and isolating multiple types of tumor cells," ACS Nano, vol. 5, no. 2, pp. 761-770, 2011.

[12] S. A. Martel-Estrada, I. Olivas-Armendáriz, E. Santos-Rodríguez et al., "Evaluation of in vitro bioactivity of Chitosan/Mimosa tenuiflora composites," Materials Letters, vol. 119, pp. 146149, 2014.

[13] C. C. Gonzalez, C. A. M. Pérez, A. M. Martínez et al., "Development of antibody-coated magnetite nanoparticles for biomarker immobilization," Journal of Nanomaterials, vol. 2014, Article ID 978284, 7 pages, 2014. 
[14] D. Lago-Cachón, M. Rivas, C. López-Larrea, A. López-Vázquez, G. Martínez-Paredes, and J. A. García, "HeLa cells separation using MICA antibody conjugated to magnetite nanoparticles," Physica Status Solidi (c)_Current Topics in Solid State Physics, vol. 11, no. 5-6, pp. 1043-1047, 2014.

[15] B. A. Otieno, C. E. Krause, and J. F. Rusling, "Bioconjugation of antibodies and enzyme labels onto magnetic beads," Methods in Enzymology, vol. 571, pp. 135-150, 2016.

[16] A. K. Hauser, R. Mathias, K. W. Anderson, and J. Zach Hilt, "The effects of synthesis method on the physical and chemical properties of dextran coated iron oxide nanoparticles," Materials Chemistry and Physics, vol. 160, pp. 177-186, 2015.

[17] X. Liu, J. Xing, Y. Guan, G. Shan, and H. Liu, "Synthesis of amino-silane modified superparamagnetic silica supports and their use for protein immobilization," Colloids and Surfaces A: Physicochemical and Engineering Aspects, vol. 238, no. 1-3, pp. 127-131, 2004.

[18] K. Can, M. Ozmen, and M. Ersoz, "Immobilization of albumin on aminosilane modified superparamagnetic magnetite nanoparticles and its characterization," Colloids and Surfaces B: Biointerfaces, vol. 71, no. 1, pp. 154-159, 2009.

[19] R. T. Reza, C. A. M. Pérez, C. A. R. González, H. M. Romero, and P.E. G. Casillas, "Effect of the polymeric coating over Fe3O4 particles used for magnetic separation," Central European Journal of Chemistry, vol. 8, no. 5, pp. 1041-1046, 2010.

[20] I. A. Flores-Urquizo, P. García-Casillas, C. Chapa-González, and P. García-Casillas, "Development of magnetic nanoparticles $\mathrm{Fe}_{2}^{+3} \mathrm{X}^{+2} \mathrm{O}_{4}(\mathrm{X}=\mathrm{Fe}$, Co y Ni) coated by amino silane," Revista Mexicana de Ingeniería Biomédica, vol. 38, no. 1, 2017.

[21] H.-S. Cho, K. Mason, K. X. Ramyar et al., "Structure of the extracellular region of HER2 alone and in complex with the Herceptin Fab," Nature, vol. 421, no. 6924, pp. 756-760, 2003.

[22] R. D. M. C. Filho, P. Kassab, L. C. L. Claro et al., "Evaluation of the expression of the human epithelial receptor 2 (HER2) in gastric carcinoma," The Scientific World Journal, vol. 2016, Article ID 7951365, 6 pages, 2016.

[23] R. J. Morrow, N. Etemadi, B. Yeo, and M. Ernst, "Challenging a misnomer? The role of inflammatory pathways in inflammatory breast cancer," Mediators of Inflammation, vol. 2017, Article ID 4754827, 15 pages, 2017.

[24] S. Loibl and L. Gianni, "HER2-positive breast cancer," The Lancet, vol. 389, no. 10087, pp. 2415-2429, 2017.

[25] J. O. Eloy, R. Petrilli, D. L. Chesca, F. P. Saggioro, R. J. Lee, and J. M. Marchetti, "Anti-HER2 immunoliposomes for co-delivery of paclitaxel and rapamycin for breast cancer therapy," European Journal of Pharmaceutics and Biopharmaceutics, vol. 115, pp. 159-167, 2017.

[26] S. Parakh, H. K. Gan, A. C. Parslow, I. J. G. Burvenich, A. W. Burgess, and A. M. Scott, "Evolution of anti-HER2 therapies for cancer treatment," Cancer Treatment Reviews, vol. 59, pp. 1-21, 2017.

[27] von Minckwitz G. et al., "Adjuvant pertuzumab and trastuzumab in early HER2-positive breast cancer," The New England Journal of Medicine, vol. 377, no. 7, pp. 702-702, 2017.

[28] X. X. Han, B. Zhao, and Y. Ozaki, "Surface-enhanced Raman scattering for protein detection," Analytical and Bioanalytical Chemistry, vol. 394, no. 7, pp. 1719-1727, 2009.

[29] P. Scherrer, in Bestimmung der inneren Struktur und der Größe von Kolloidteilchen mittels Röntgenstrahlen, Kolloidchemie Ein Lehrbuch, pp. 187-409, Springer, Berlin, Germany, 1912.

[30] S. A. Majetich and Y. Jin, "Magnetization directions of individual nanoparticles," Science, vol. 284, no. 5413, pp. 470-473, 1999.
[31] D. Han, S. C. Hong, J. H. Lee et al., "Subtle cytotoxicity and genotoxicity differences in superparamagnetic iron oxide nanoparticles coated with various functional groups," International Journal of Nanomedicine, vol. 6, pp. 3219-3231.

[32] C. Marcott, M. Padalkar, and N. Pleshko, "Infrared and raman microscopy and imaging of biomaterials at the micro and nano scale," in Comprehensive Biomaterials II, pp. 498-518, Elsevier, 2017.

[33] E. Alp and N. Aydogan, "A comparative study: synthesis of superparamagnetic iron oxide nanoparticles in air and $\mathrm{N}_{2}$ atmosphere," Colloids and Surfaces A: Physicochemical and Engineering Aspects, vol. 510, pp. 205-212, 2016.

[34] L. V. Gasparov, D. Arenas, K. Choi et al., "Magnetite: Raman study of the high-pressure and low-temperature effects," Journal of Applied Physics, vol. 97, no. 10, p. 10A922, 2005.

[35] P. Panta and C. Bergmann, "Raman spectroscopy of iron oxide of nanoparticles (Fe3O4)," Journal of Material Science \& Engineering, vol. 05, no. 01, pp. 1-3, 2015.

[36] N. Lee, P. J. Schuck, P. S. Nico, and B. Gilbert, "Surface enhanced Raman spectroscopy of organic molecules on magnetite (Fe3O4) nanoparticles," The Journal of Physical Chemistry Letters, vol. 6, no. 6, pp. 970-974, 2015.

[37] M. A. G. Soler and F. Qu, "Raman spectroscopy of iron oxide nanoparticles," in Raman Spectroscopy for Nanomaterials Characterization, pp. 379-416, Springer, Berlin, Germany, 2012.

[38] R. Tuma, "Raman spectroscopy of proteins: from peptides to large assemblies," Journal of Raman Spectroscopy, vol. 36, no. 4, pp. 307-319, 2005.

[39] H. Takeuchi, "Raman structural markers of tryptophan and histidine side chains in proteins," Biopolymers-Biospectroscopy Section, vol. 72, no. 5, pp. 305-317, 2003. 


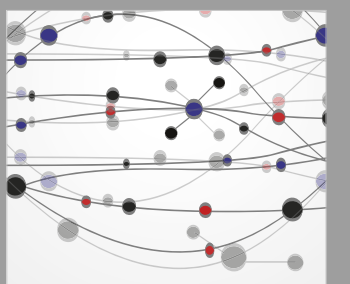

The Scientific World Journal
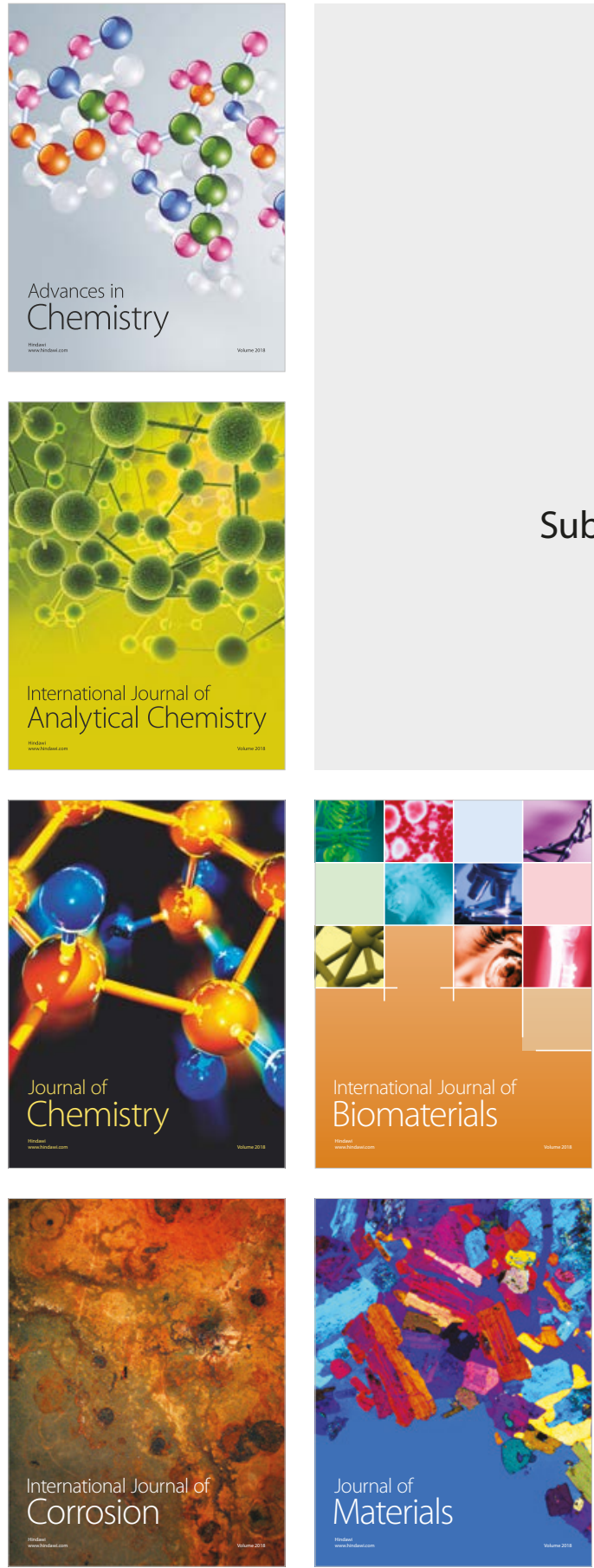

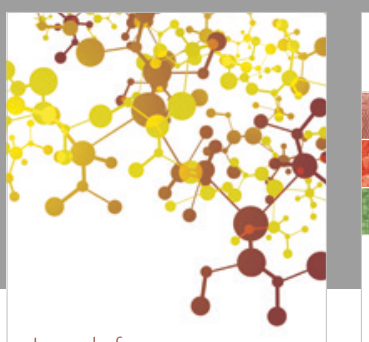

Journal of

Applied Chemistry
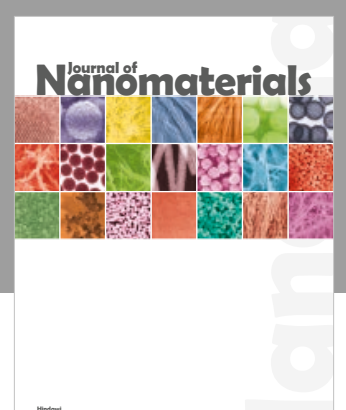

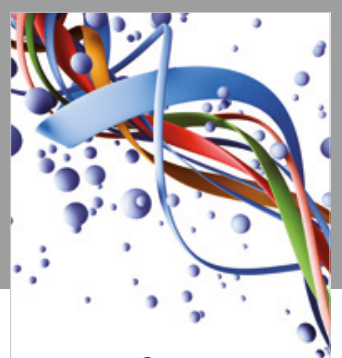

Scientifica

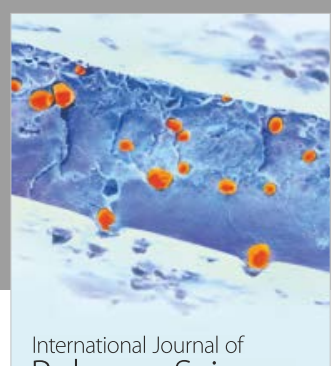

Polymer Science

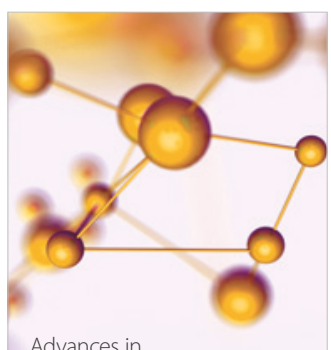

Physical Chemistry
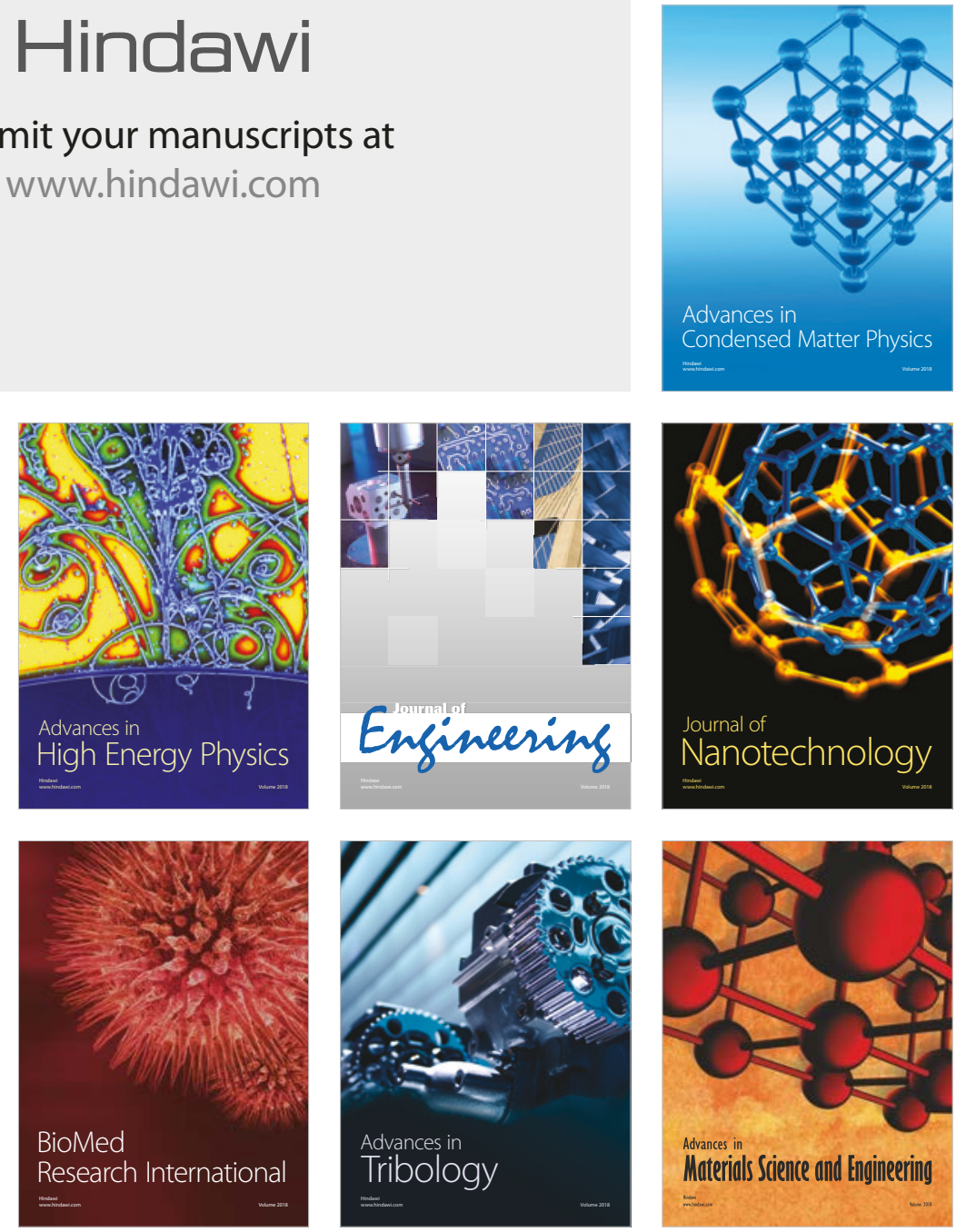